\section{3-D Reconstruction of Thick IVEM Samples Using Tuned Aperture Computed Tomography® (TACT®)}

W. Gray Jerome, Ken Grant, Patricia G. Yancey,

A. M. Al Gailany, Wolfram Betterman, Richard L. Webber Wake Forest University School of Medicine

Most objects in our world are 3-dimensional (3-D), and this is certainly true of celluiar ultrastructure. The challenge in microscopy has always been how to analyze this 3-D information. Traditional thin-section microscopy has a minimal ability to view 3-D structure, because it is limited to viewing thin, almost twodimensional (2-D) planes taken from the specimen. The development of high and intermediate voltage electron microscopes (HVEM and IVEM) provides the ability to investigate the ultrastructure of thick biological samples-allowing a unique view of the 3-D interrelationships of cells and organelles.

In a single view of thick material, however, we still view only a 2-D projection of the 3-D object. We superimpose all information along the depth or axial dimension ( $Z$ axis). At least two images from different perspectives are required for us to begin to analyze depth information. Thus, analysis and display of the 3-D information obtained from higher voltage microscopes have presented challenges, particularly to quantitative biologists. Advances in adapting computed tomography (CT) to electron microscopy have provided the major means of quantitative 3-D analysis of cellular ultrastructure ${ }^{1,2}$. CT uses images from multiple different tilt angles to construct a quantitative map of the original material and can provide reasonably highresolution results. In particular, tremendous gains in determin- ing the structure of macromolecules have occurred with advances in electron microscopy $\mathrm{CT}^{3}$. However, high resolution, backprojection $\mathrm{CT}$ requires a very large number of tilt angles to be sampled and usually requires high end computers for efficient production of true 3-D reconstructions.

We have adapted an alternative approach, Tuned Aperture Computer Tomography® (TACT®), for use with IVEM and HVEM and even with scanning electron microscopy (SEM). TACT is similar to $\mathrm{CT}$ in that it uses a series of images of the sample taken from different angles. However, unlike CT, useable TACT reconstructions can be obtained from an arbitrarily selected number of oblique projections exposed from any desired distribution of multiple angles. This capability makes reconstruction of beam-sensitive material possible. The most common form of TACT output is a series of parallel slices through the specimen along the optical axis. This type of display is similar to that produced from a confocal microscope, and, like a confocal reconstruction, the full resolution of the sample along the $X-Y$ plane is maintained. In addition, programs designed to analyze confocal data stacks can be used directly to analyze TACT data stacks.

As in classic tomosynthesis ${ }^{4}$, TACT uses simple backprojection to produce tomographic slices. A data set is obtained by taking a series of images of a sample from an arbitrarily selected number and distribution of tilt angles. The quality of the reconstruction is determined by the information content of the input images, the angular disparity of the projections, and the total number of angles sampled. In general, axial resolution improves with greater angular spread between projections, and the signalto-noise ratio (SNR) improves with relatively larger numbers of projections. TACT reconstruction is accomplished by using the
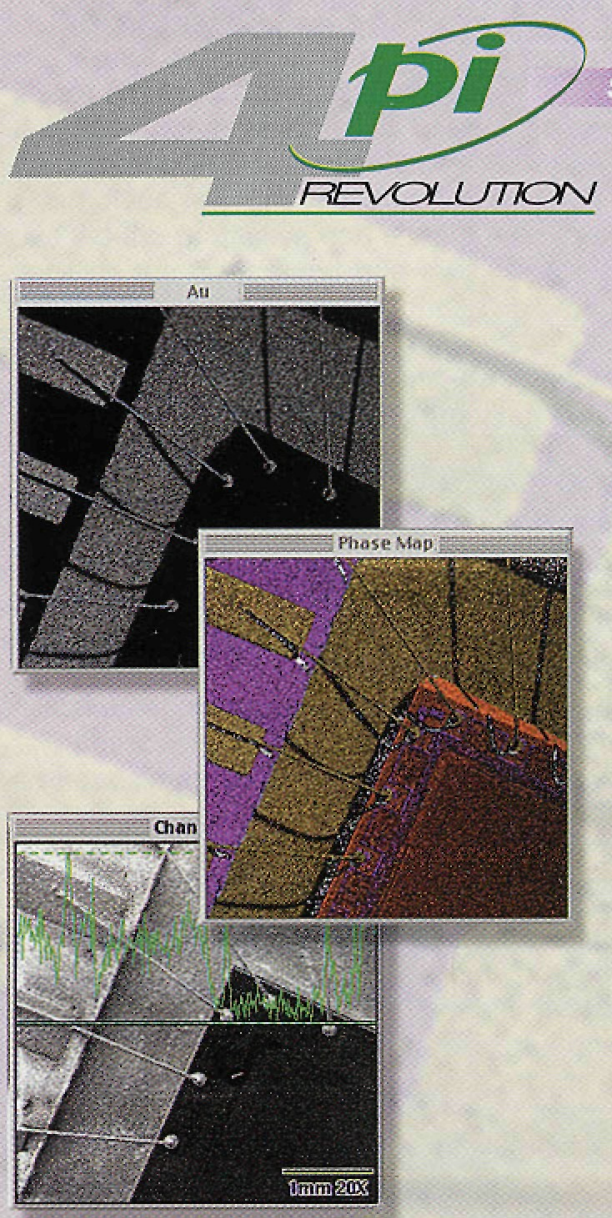

Soectral and lmage Acquisition software for Windows ${ }^{\circ}$ and Maco OS

\section{- Micron markers}

- Easy-to-use point-and-click screen tools

- Preview and acquire images of different sizes

- Multiple spectra windows with independent scaling and colors

- Simultaneous acquisition of SEM, WDS, SCA and X-ray images

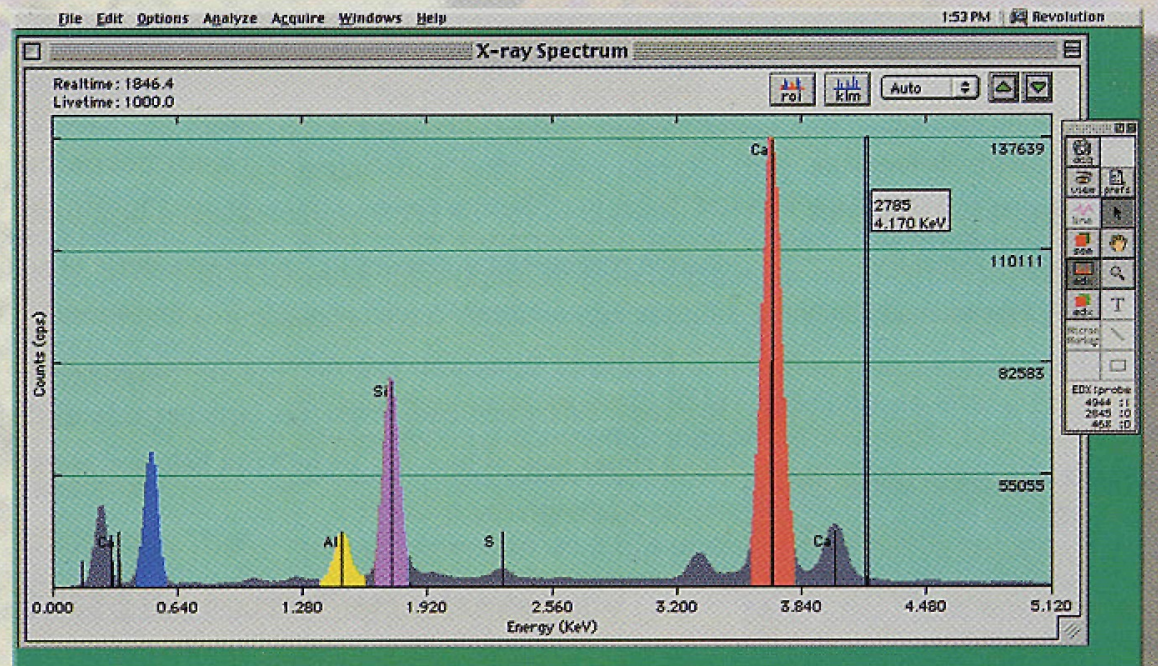

4 pi Analysis, Inc. X-ray detectors, EDS and Imaging Systems and Upgrades (919) $489-1757$

sales@4pi.com •www.4pi.com 
same set of recognizable details ("fiducial references") in each projection to provide functional relationships that permit appropriate registration and projective transformation of all of the original projections. Simply shifting and adding these transformed projections by selected amounts permit $3 \mathrm{D}$ resolution of objects into homologous focal planes. For example, after all of the appropriate projective transformations have been accomplished based on the respective tilt angles selected, all that is required to generate a new focal plane is a proportional shift of each projection image either toward or away from a single arbitrary point of convergence. Two of the advantages of TACT, besides its ease of use and relative simplicity, are that it requires no a priori information about the

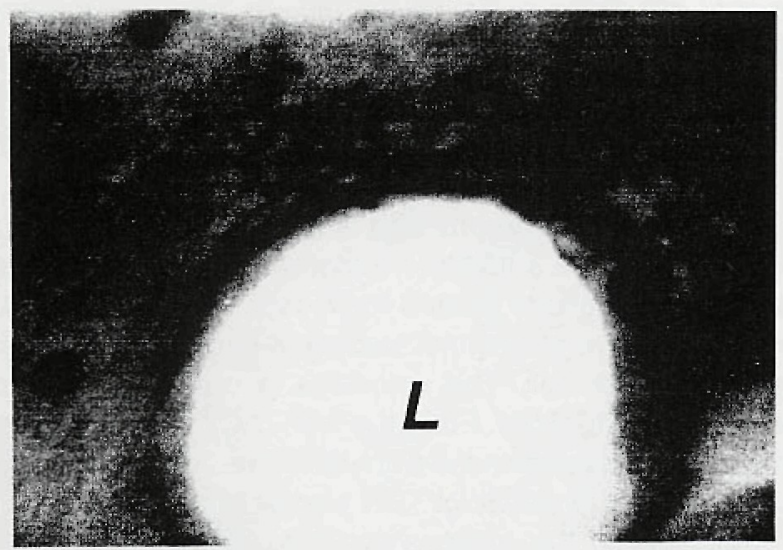

Figure 1. IVEM image of acid phosphatase stained smooth muscle cell. The section is approximately $3 \mu \mathrm{m}$ in thickness. The dark stain highlights the periphery of a large lipid filled lysosome $(\mathrm{L})$ in the lower center of the image and shows the an extensive array of Golgi/TGN tubules capping the lipid droplet sample and it can be tuned to the resolution required. For beamsensitive material, it is possible either to prorate the energy across multiple projections or to settle for relatively fewer projections. The respective consequences are reconstructions having reduced SNRs in the former situation and less uniform suppression of blurring artifacts in the latter. A typical reconstruction requires less than half a day to complete.

TACT, like circular tomosynthesis ${ }^{4}$, does not provide complete independence of axially-oriented slice information ${ }^{5}$. Hence, depth resolution necessarily is degraded by artifacts produced from out-of-focus structures. However, the effects of these artifacts can be mitigated with well-known deblurring methods, such as iterative deconvolution. Consequently, TACT can be applied to

\section{Continued on following page}

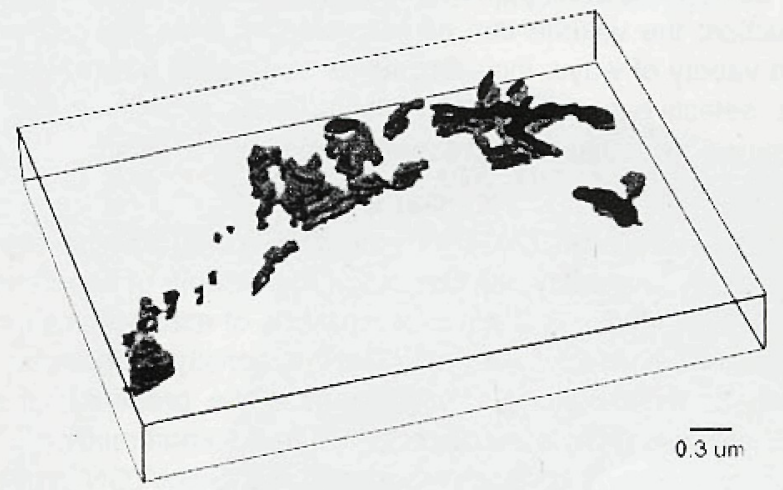

Figure 5. Psuedohologram reconstruction of the sample in figure 3 . The reconstruction was formed by stacking all of the sequential TACT slices.

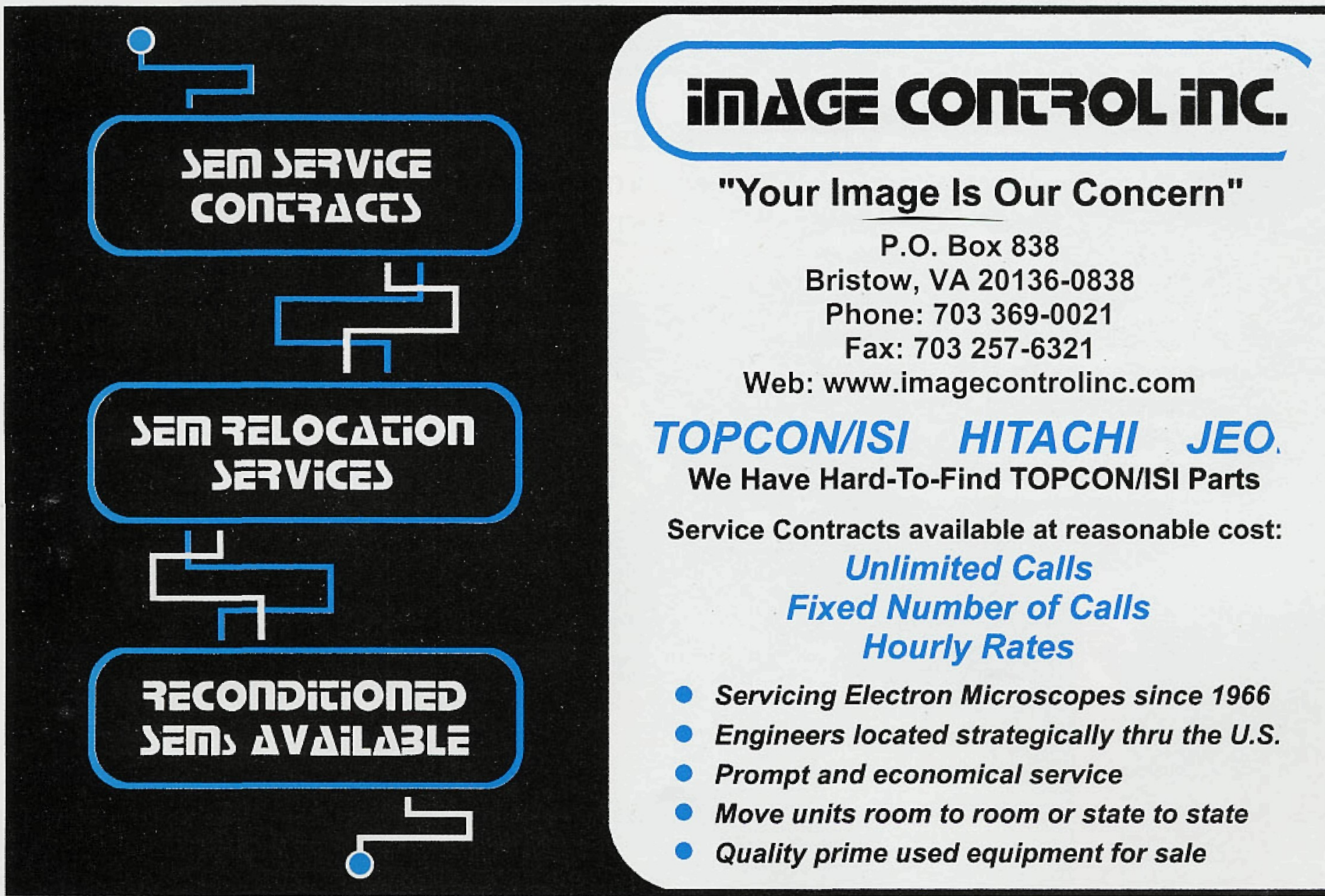




\section{3-D Reconstruction of Thick IVEM Samples Using Tuned Aperture Computed Tomography (TACT) \\ Continued from proceeding page}

a variety of applications constrained by projection limitations that otherwise would preclude the straight-forward application of $C T$, such as $x$-ray breast imaging ${ }^{6}$.

TACT output can be displayed as a series of images representing parallel "slices" through the volume. These slices can be oriented in any direction but the image quality generally decreases when the normal to the slice plane diverges significantly from the axial direction. TACT slices can be analyzed individually or reconstituted to produce a dynamic holographic type of 3D display. Quantitation of structures within the sample is simply a matter of summing a series of $2 \mathrm{D}$ measures made on each computed planar slice. Following the initial reconstruction, the volume can be processed further and presented in a variety of ways, including full or segmental volume rendering, selective component pseudocoloring, or convex hull delineation, depending upon available external software.

\section{Methods}

We have used TACT for quantitative 3-D IVEM in several studies. In one study, we computed the volume of hypertrophic Golgi/TGN stacks in $3 \mu \mathrm{m}$ thick sections of macrophages incubated for seven days with oxidized low density lipoprotein. The samples were stained to demonstrate the presence of acid phosphatase using a modification ${ }^{7}$ of the Gomori method ${ }^{8}$. This highlighted the lipid-filled lysosomes and Golgi/TGN region of the cells. The thick sections of epoxy embedded material were viewed by IVEM (Philips CM30) at $300 \mathrm{KeV}$ and images cap- tured with a Gatan 622 fiber optic TV camera. For the reconstruction shown here, nineteen images were used, representing images taken from +45 degrees of tilt to -45 degrees in 5 -degree increments. Only two fiducial references were required to implement a self-calibrating scheme of image registration. For clarity, the stained Golgi/TGN tubules were converted to geometric primitives using thresholding techniques, and the 3-D reconstructions were computed using the TACT program and displayed using VoxBlast $^{\text {TM }}$ software (Vaytek, Inc.). The TACT and VoxBlast algorithms were performed on a PC operating under Windows NT 4.0.

In related experiments, TACT displays were made of SEM samples containing cholesterol crystals. One such display is included in this manuscript. Digital images were captured directly from a 15-degree conical tilt series of the original SEM sample with 40-degrees of rotation between each of 9 images. Two fiducial reference points were used for the reconstruction which consisted of a stack of 45 thick $(27 \mathrm{~nm})$ slices.

\section{Results and Discussion}

Figure 1 shows an IVEM micrograph of a thick $(3 \mu \mathrm{m})$ section through a macrophage treated for 7 days with oxidized LDL. This produces massive cholesterol loading. A large lipid-filled lysosome is present in the cell. This lysosome is capped by stained elements of the Golgi/TGN. From the 3-D TACT reconstruction of the acid phosphatase stained elements of the Golgi/TGN region, the volume of the stained Golgi/TGN elements was computed as 9.4 $\mu \mathrm{m}^{3}$ by summing the volumes occupied in each slice. The Golgi/ TGN image was further processed to clarify the image for presentation. Geometric primitives were computed using simple thresholding techniques and the 3-D display computed. This simplified reconstruction is seen in figure 2 .

\section{Si(Li)-Detectors}

stainless steel dewar, UHV technology excellent energy resolution best choice for light element analysis unrivalled long term stability

\section{Microanalysis Systems}

standardless analysis on rough surfaces and particles physical calculation of the bremsstrahlung -integrated wexpert systemk comprehensive error calculation application-specific automatic quantification
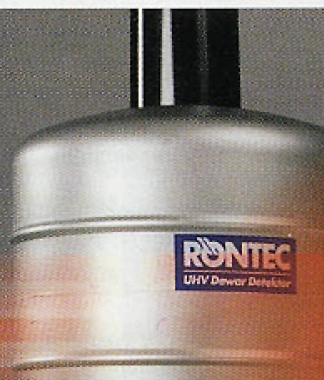

NEW!

Element Imaging

liquid nitrogen free RÖNTEC XFlash', detecto

8 times foster than EDX

with digital pulse processing

combines with any existing EDX

50

30
kcps or imaging system

poras


Although primarily designed for reconstruction of transparent specimens, TACT is also adaptable to the 3-D display and measurement of solid objects, such as those viewed by SEM. Figure 3 shows an SEM of macrophages in culture in the center of which is a large cholesterol crystal formed by the surrounding cells. Figure 4 shows a single slice image from the TACT reconstruction of the crystal in which the top of the crystal is in focus but the cells below are blurred. Obviously TACT is not required to provide a 3$\mathrm{D}$ surface rendering image of the crystal because it is provided by the SEM image itself. However, for a true 3-D image that can be visualized from multiple angles or which can be seen as a series of relative elevations that facilitate quantition of crystal size and volume, more than one projection is required. By using the TACT reconstruction we estimate the volume of the crystal as $1149 \mu^{3}$.

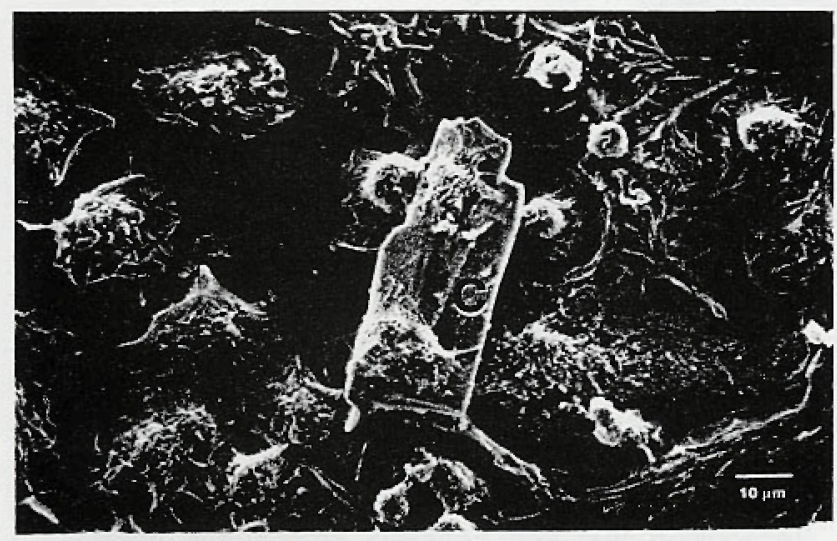

Figure 3. SEM image of macrophages and a large cholesterol crystal (C) formed by the macrophages.

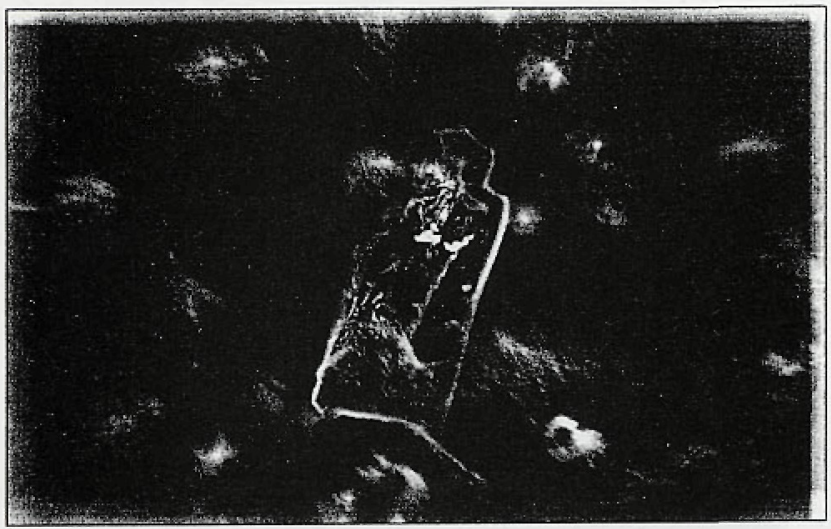

Figure 4. Single slice from the reconstruction of the sample in figure 3 . Note that the top of the crystal is in focus but bottom of the crystal and the macrophages, which are below the plane of the slice, are out of focus.

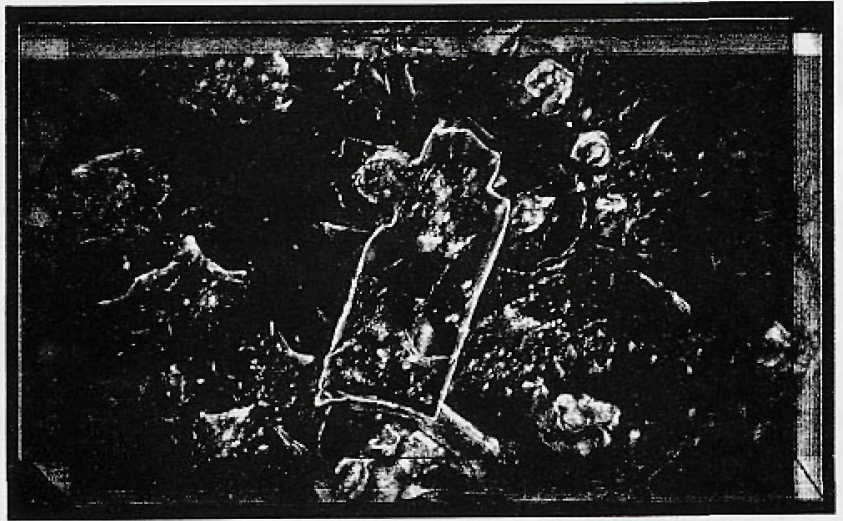

Figure 5. Psuedohologram reconstruction of the sample in Figure 3. The reconstruction was formed by stacking all of the slices.
Figure 5 shows a pseudohologram rendering of the reconstruction presenting all the slices stacked together.

In summary, the advantages of TACT are its ease of implementation, the fact that it requires no a priori information about the sample, and that it can be tuned to the 3-D image quality required. This increases the efficiency of effort. Running on simple desk top PCs, a typical reconstruction requires less than a day to complete including image collection. It thus offers a viable alternative to EM computed axial tomography in situations where the cost requirements or the need for rapid quantitation of multiple samples outweighs the necessity for complete slice independence.

1. Perkins, G. A., C. Renken, J. Song, T. Frey, S. Young, S. Lamont, M. Martone, S. Lindsey, and M. Ellisman. 1997. Electron tomography of large, multicomponent biological structures. J Struct Biol. 120:219-27

2. Koster, A., R. Grimm, D. Typke, R. Hegerl, A. Stoschek, J. Walz, and W. Baumeister. 1997. Perspectives of molecular and cellular electron tomography. J Struct Biol. 120:276-308.

3. Frank, J. 1996, Three-dimensional electron microscopy of macromolecular assemblies, Academic Press, San Diego.

4. Grant, D. G. 1972. Tomosynthesis: a three-dimensional radiographic imaging technique. IEEE Trans Biomed Engineering. 19:20-28.

5. Webber, R., R. Horton, D. Tyndall, and J. Ludlow. 1997. Tunedaperture computed tomography (TACT). Theory and application for three-dimensional dentoalveolar imaging. Dentomaxillifacial Radiol. 26:53-62

6. Webber, R., H. Underhill, and R. Freimanis. 2000. A controlled evaluation of tuned-aperture computed tomography applied to digital spot mammography. J Digit Imaging. 13:1-9.

7. Jerome, W. G., and J. C. Lewis. 1985. Early atherogenesis in White Carneau pigeons II Ultrastructural and cytochemical observations. Am J Pathol. 119:210-222.

8. Gomori, G. 1950. An improved histochemical technic for acid phosphatase. Stain Technol. 25:81-85.

* TACT is copyrighted by Wake Forest University School of Medicine. TACT requires a 486 or better $P C$ running Windows $(95$, NT 4.0 , or higher) and sufficient RAM to handle large image files. The program is still in the developmental stages. Anyone interested in working with the program for research purposes or in commercial applications is encouraged to contact Dr. Jay Jerome (jjerome@wfubmc.edu) or Wake Forest University's Technology Asset Management Office (in care of Dr. William H. Williams, Wake Forest University School of Medicine, Medical Center Blvd., Winston-Salem, NC 27157

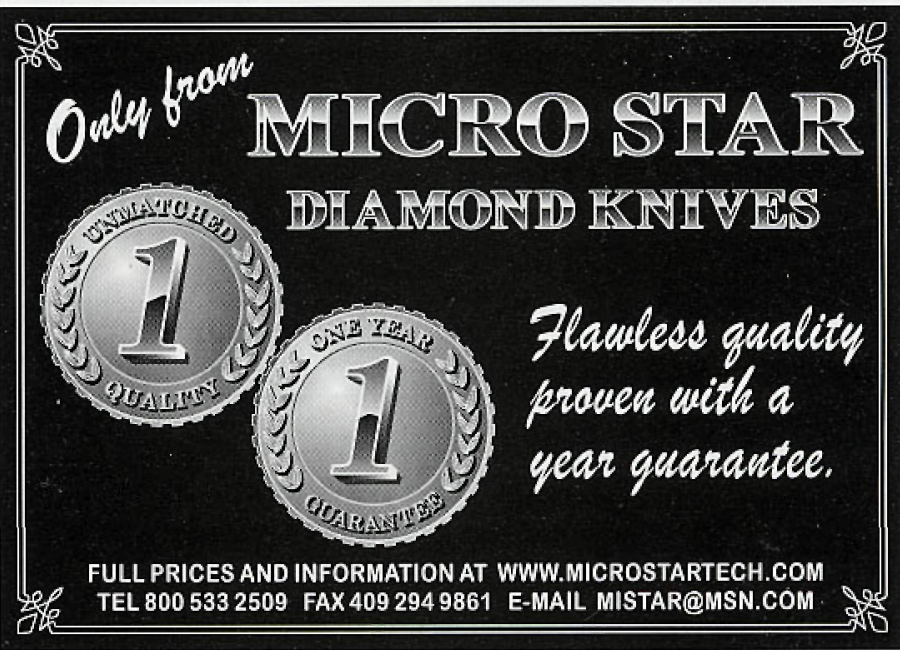

